\title{
Oxidative stress and periodontal disease in diabetic patients: a 3-month pilot study
}

\author{
Simone Marconcini ${ }^{*}$, Enrica Giammarinaro ${ }^{2}$, Ottavio Giampietro ${ }^{3}$, Chiara Giampietro ${ }^{3}$, Birgitta Soder ${ }^{4}$, Annamaria Genovesi Rdh ${ }^{2}$, \\ Antonio Barone ${ }^{1,2}$ and Ugo Covani ${ }^{1,2}$ \\ ${ }^{1}$ Department of Surgical, Medical, Molecular and Critical Area Pathology, University of Pisa, Italy \\ ${ }^{2}$ Tuscan Dental Institute, Foundation for Dental Clinic, Research and Continuing Education, Versilia General Hospital, Lido di Camaiore (LU), Italy \\ ${ }^{3}$ Department of Clinical and experimental medicine, University-Hospital at Pisa, Italy \\ ${ }^{4}$ Department of Dental Medicine, Karolinska Institute, Stockholm, Sweden
}

\begin{abstract}
Aim: Oxidative stress is involved in both Periodontal Disease (PD) and Diabetes Mellitus (DM). The present study aimed to evaluate the oxidative balance in diabetic patients diagnosed with PD before and after non-surgical periodontal treatment.

Materials and methods: Sixty patients were divided into three groups all receiving non-surgical periodontal treatment plus either chlorhexidine, ozone-therapy or antioxidant mouth-rinse. Probing depth (PPD), Percentage Plaque Index (\%PI) and Percentage Bleeding on Probing (\%BoP) were recorded. Free-radicals (dROMs), plasmatic antioxidants (PAT), salivary antioxidants (SAT) and glycated hemoglobin ( $\mathrm{HbA}_{1} \mathrm{c}$ ) were measured. Results: Mean PPD at baseline was $3.14 \mathrm{~mm}$. Mean PPD three months after was $2.05 \mathrm{~mm}$. The overall $\% \mathrm{PI}$ at baseline was $55 \%$ and $\% \mathrm{BOP} 76 \%$. Three months after treatment $\% \mathrm{PI}$ was $34 \%$ and $\% \mathrm{BoP}$ was $64 \%$. The longitudinal analysis did not show differences between groups. Mean dROMs at baseline was $353 \mathrm{U}$. Carr (oxidative stress) and decreased after three months reaching 295 U. Carr (normal). SAT was $2083 \mathrm{U}$. Carr at baseline (inflammation) and decreased to 1337 U. Carr (ideal). The longitudinal analysis did not show differences between groups. Mean $\mathrm{HbA}_{1} \mathrm{c}$ at baseline was $6.92 \%$ and decreased significantly to $6.63 \%$ three months after treatment.
\end{abstract}

Conclusion: Based on the results of the present study, oxidative stress should be further investigated as a potential modulator of the clinical course of both DM and PD.

\section{Introduction}

Periodontal Disease is caused by a dysbiosis, an imbalance in the relative presence or influence of microbial species that take part of the oral microbiome [1,2]. The dysbiotic microbial community, if left untreated, might cause or, at least, exacerbate other systemic inflammatory disorders [3]. In the last decades, many researchers have focused on the association between periodontitis and various systemic inflammatory pathologies. An increasing number of studies have suggested that systemic inflammation might sometimes result from the entry of oral microbial agents and their virulence factors into the circulation [4].

Diabetes mellitus (DM) is increasingly common worldwide [5]. Diabetic subjects present an increased prevalence and severity of periodontitis [6,7]. The pathological microbial environment of periodontitis has been suggested as a metabolic influencer $[8,9]$. The nature of the relationship is still debated [10]. Several mechanisms have been proposed to explain it: increased oxidative stress, advanced glycation end-products, altered immune function, and changes in collagen. Nowadays, there is a broad consent about the fact that periodontal treatment might improve glycaemic control: a significant reduction of $\mathrm{HbA}_{1 \mathrm{C}} 3$ months after periodontal treatment, has been demonstrated [11-13]. Since periodontitis is greatly untreated, what measures would improve its management in persons at risk for complications of diabetes? Many inflammatory chemokines and endotoxins (c-reactive protein, interleukin 6 , tumour necrosis factor a) have been investigated as markers of systemic inflammation in diabetic patients [14]. Lately, a large amount of literature has been directed to the study of reactive oxygen species and their role in the pathophysiology of different systemic inflammatory disorders [15]. Oxidative stress is caused by an excessive release of free radicals or by a deficiency of anti-oxidant agents. A great amount of pro-oxidant agents is produced during inflammatory conditions, such as chronic periodontal disease [16]. The overexposure to free radicals may worsen systemic inflammatory conditions leading to tissue damage [17]. Saliva is rich in molecules, such as uric acid, vitamin $\mathrm{C}$, reduced glutathione (GSH), oxidized glutathione (GSSG), lacto-ferrine, and many others [18]. Since those molecules work in concert renewing each other, total antioxidant capacity may be useful to assess individuals 'defense capabilities. The aim of the present prospective clinical study was to investigate the putative correlation between oxidative stress, clinical periodontal indexes and glycaemic status in diabetic patients. The null hypothesis was that non-surgical periodontal treatment does not induce any remarkable difference in terms of markers of oxidative stress and glycaemic status.

Correspondence to: Simone Marconcini, DDS, PhD, MSc, Department of Surgical, Medical, Molecular and Critical Area Pathology, University of Pisa, Via Roma 55, 56100 Pisa (PI), Italy, Tel: +39 (0584) 6059888; Fax: +39 (0584) 6058716, E-mail: simosurg@gmail.com

Key words: periodontal disease, oxidative stress, salivary antioxidant capacity

Received: May 01, 2017; Accepted: May 12, 2017; Published: May 15, 2017 


\section{Materials and methods}

Sixty patients were recruited for this prospective randomized clinical study. All of the patients were diagnosed with Diabetes Mellitus (DM) type 1 or 2 at the Diabetology Department of the University of Pisa. Detailed information of their current medications were collected as well as their glycemic control status.

Each patient had to sign an informed written consent and to answer a specific anamnestic questionnaire.

The study has been conducted in accordance with the Helsinky declaration of 2002 .

\section{Selection criteria}

Inclusion criteria for study participation were an age range of 1870 years and the diagnosis of both Diabetes Mellitus (type 1 and type 2) and Periodontitis.

\section{Exclusion criteria were}

Pregnancy, conditions requiring antibiotic prophylaxis, psychiatric disorders, severe physical handicaps, carcinoma, immunosuppressive therapy, hospital in-patient, vitamins supplements or antibiotics taken within 3 months before the study or periodontal therapy received within 6 months.

\section{Study design}

The study design was a prospective clinical study. The cohort size was determined by power calculation analysis according to previous published literature. Recruitment began in September 2015, and the follow-up is still on-going. The last recall visit is due to be within 12 months from baseline. All of the recruited patients underwent a periodontal examination (clinical assessment with a standard periodontal UCLA probe) and were eventually asked to participate in the experimental part of the study. Diagnosis of periodontal disease was assessed as the proximal attachment loss $\geq 3 \mathrm{~mm}$ in $\geq 2$ non-adjacent, BOP on at least $25 \%$ of their total sites and documented radiographic bone loss [19].

At baseline $\left(\mathrm{T}_{0}\right)$ patients were randomly allocated to one of the three groups of treatment, so that a professional hygiene session was performed and an adjunctive domiciliary therapy was prescribed. At the same time, clinical and laboratory parameters were collected. The follow-up schedule comprised two recall visits: one month $\left(\mathrm{T}_{1}\right)$ and three months after periodontal treatment $\left(\mathrm{T}_{2}\right)$. Each visit consisted in a full periodontal examination and in the collection of saliva and blood samples in order to evaluate specific markers.

\section{Periodontal assessment}

One investigator performed full periodontal examinations. Periodontal probing depth (PPD) was measured using a UNC-PCP15 (Hu Friedy) probe at six sites (mesio-buccal, mid-buccal, disto-buccal, mesio-lingual and disto-lingual) per tooth (third molars excluded). Mean values of PPD per patient were calculated. Marginal bleeding on probing (BoP) and plaque index (PI) were assessed dichotomously (" 1 " present, "0" absent) at six sites per tooth. For each patient BoP and PI were then expressed as a percentage (BoP\%, PI\%) of positive sites.

\section{Periodontal treatment and motivation}

Randomization was obtained by a virtually-generated list. Diabetic patients were then assigned to one of the three groups of treatments (twenty patients per group):
- Control group: standard non-surgical periodontal treatment (ultrasonic debridement and manual scaling) plus a domiciliary prescription of a commercial chlorhexidine mouthwash for the following 7 days.

Test group: standard non-surgical periodontal treatment (ultrasonic debridement and manual scaling) plus a domiciliary prescription of an anti-oxidant mouthwash for the following 7 days.

Test group: standard non-surgical periodontal treatment (ultrasonic debridement and manual scaling) plus professional ozone administration and the domiciliary usage of an ozone delivery device (Aquolab s.r.l. ${ }^{+}$.

The ultrasonic device used was the Mectron Multipiezo (Mectron s.p.a. ${ }^{\star}$ ). The ozone delivery device was a hydro-pulseur (Aquolab s.r.l) with a magnetic drive pump that produces a continuous release of ozonized-water. Professional usage was set at a higher range of ozone tension $(12 \mathrm{~V})$ with the smallest nozzle $(0.6 \mathrm{~mm})$. Domiciliary devices were calibrated and then handed to patients. Ozone tension was set at $12 \mathrm{~V}$ and the nozzle of choice was the largest available.

Motivation to proper oral hygiene had the duration of 30 to 60 minutes per patient; the operator informed each patient about the correct maneuvers to perform at home in order to achieve the best results in terms of personal oral hygiene.

\section{Laboratory analysis}

Clinical samples were collected early in the morning. Both saliva and blood samples were processed according to instructions furnished the company producing the collection kit and the dedicated spectrophotometer (H\&D s.r. $\left.l^{\mathrm{c}}\right)$.

The d-ROM (derived reactive oxygen molecules) test determines the concentration of hydro-peroxides in the blood. Its unit of measurement is the U. CARR $(0.08 \mathrm{mg} / \mathrm{dL}$ of a solution of hydrogen peroxide). The PAT (plasmatic antioxidant test) determines the concentration of the water-soluble antioxidants in the blood that are able to reduce ferric ions to ferrous ions. Its unit of measurement is the U. CARR as well. The SAT (salivary antioxidant test) evaluates the salivary total antioxidant capacity. Saliva was immediately analyzed since it degenerates fast altering the absorbance properties of the sample.

\section{Glycemic status and medical anamnesis}

At baseline, information regarding physical activity, diet, major complications of diabetes, body mass index and smoking habits were recorded. In cooperation with the Department of Diabetology, the level of glycated hemoglobin was assessed before treatment. At three months, the new value/ post-treatment was registered.

\section{Statistical analysis}

Clinical and laboratory parameters were inserted into an Excel data-sheet. Outcome variables were defined as: mean probing depth (PPD), mean plaque index in percentage (PI\%), mean bleeding on probing in percentage (BoP\%), mean dROMs, mean PAT, mean $\mathrm{SAT}$, mean $\mathrm{HbA}_{1 \mathrm{c}}$. Descriptive and statistical analyses of data were performed by usage of two statistical tool packages (Stata ${ }^{\#}$ and $\mathrm{R}$ Studio ${ }^{\circ}$. Normal distribution was tested with the Shapiro-Wilcoxon method. All measurements in the text and Tables are described as mean and standard deviations ( $\mathrm{m} \pm \mathrm{std}$ ). Intergroup and intragroup longitudinal analysis was performed using the Brunner and Langer model for non-parametric longitudinal designs [20]. The inter-group analysis (between subject) was based on the stratification for the group 
of treatment (whole-plot factor). The intra-group analysis (within subjects) relied on the stratification for repeated measures on one subplot factor: time. The mixed method applied for longitudinal analysis consisted of Wald type statistic (WTS), ANOVA type statistic (ATS) and modified ANOVA-Type Statistic for the Whole-Plot Factors. The significance was set at $95 \%$ for all of the tests performed.

\section{Results}

A total of 75 Type 1 and 2 diabetes patients were screened; 11 patients were excluded based on their medical history and 4 for personal reasons, leaving a total of 60 patients eligible for study participation. Table 1 reports the demographic characteristics of the initial cohort. The cohort presented 22 females and 38 males; mean age was 60.9 years old with Type-I patients, being significantly younger than type II. Mean body mass index was 27.8 , being higher among type 2 Diabetes patients. Eleven patients were smokers. Twenty-two out of 60 patients suffered from DM type 1 and the left 38 patients were affected by DM type 2 .

Table 2 reports the periodontal findings for each group of treatment, for every moment of the follow-up. Mean probing depth (PPD) for the entire cohort at baseline was $3.14 \mathrm{~mm}$. The overall mean plaque index at baseline was $55 \%$. Bleeding on Probing (BOP) resulted positive in $76 \%$ of sites. Three months after treatment, the global mean of PPD was $2.05 \mathrm{~mm}$. The mean percentage plaque presence decreased to $34 \%$ of positive sites. Bleeding on Probing showed a positive percentage at 3 months of $64 \%$ of sites analyzed. The oxidative balance descriptive statistics is reported in table 3 . The mean amount of plasmatic free radicals (d-ROM) for the cohort at baseline was 353 U.Carr. which is higher than the mean values of the general population. Three months after periodontal non-surgical treatment the mean value recorded was 295 U.Carr. which is considered to be in a normal range. The mean concentration of Plasmatic Antioxidant agents at baseline was 1838 U.Carr. which is considered a deficiency status. Coming to the analysis of Total Antioxidant Capacity of Saliva at baseline the mean value was 2083 U.Carr which is considered a high value denoting periodontal inflammation. Three months after treatment mean SAT for the entire cohort was 1337 U. Carr, a value that is considered ideal.

The longitudinal intra- and inter-group analysis is reported in table 4. Time was set as a sub-plot factor (intragroup analysis or within subjects) and the group of treatment was fixed as a whole-plot factor. When time was the only factor considered in the analysis, each clinical outcome variable changed significantly three months after treatment (within subjects). Periodontal indices of inflammation (PPD, PI\%, BoP\%) decreased significantly denoting an improvement in periodontal health after non-surgical therapy. Anyway, none of the tests applied reached statistically significant $\mathrm{p}$-values for the whole-plot factor (group of treatment) indicating that the null hypothesis of no treatment effect on periodontal status could not be rejected. Without reaching significance, Ozone group showed the best results in terms of periodontal inflammation reduction.

Table 1. Demographic characteristics.

\begin{tabular}{|c|c|}
\hline Variable & Mean \pm SD \\
\hline Number of patients & 60 \\
\hline Number of DM-1 & 22 \\
\hline Number of DM-2 & 38 \\
\hline Number of males & 38 \\
\hline Number of smokers & 11 \\
\hline Age & $60.9 \pm 2.71$ \\
\hline Body mass index & $27.8 \pm 0.90$ \\
\hline
\end{tabular}

Table 2. Mean ( \pm standard deviation) PPD, \%PI, \% BoP outcome measures at baseline, one month and three months.

\begin{tabular}{|c|c|c|c|c|c|}
\hline \multirow[t]{2}{*}{ Variable } & \multirow[t]{2}{*}{ Time point } & \multirow[t]{2}{*}{ Overall } & \multicolumn{3}{|c|}{ Treatment Group } \\
\hline & & & Clhorhexidine & Ozone & Antioxidant \\
\hline & & Mean \pm SD & Mean \pm SD & Mean \pm SD & Mean \pm SD \\
\hline \multicolumn{6}{|l|}{ PPD } \\
\hline & Baseline & $3.14 \pm 0.26$ & $3.43 \pm 0.39$ & $3.06 \pm 0.33$ & $3.35 \pm 0.27$ \\
\hline & 1 month & $2.11 \pm 0.11$ & $2.23 \pm 0.24$ & $2.60 \pm 0.22$ & $2.17 \pm 0.29$ \\
\hline & 3 months & $2.05 \pm 0.22$ & $2.25 \pm 0.49$ & $1.96 \pm 0.16$ & $2.26 \pm 0.33$ \\
\hline \multicolumn{6}{|l|}{$\% \mathrm{PI}$} \\
\hline & Baseline & $0.55 \pm 0.07$ & $0.53 \pm 0.14$ & $0.51 \pm 0.12$ & $0.65 \pm 0.16$ \\
\hline & 1 month & $0.15 \pm 0.04$ & $0.13 \pm 0.09$ & $0.17 \pm 0.08$ & $0.15 \pm 0.05$ \\
\hline & 3 months & $0.34 \pm 0.07$ & $0.45 \pm 0.12$ & $0.30 \pm 0.12$ & $0.25 \pm 0.12$ \\
\hline \multicolumn{6}{|l|}{$\% \mathrm{BoP}$} \\
\hline & Baseline & $0.76 \pm 0.10$ & $0.71 \pm 0.18$ & $0.66 \pm 0.16$ & $1.00 \pm 0.00$ \\
\hline & 1 month & $0.41 \pm 0.12$ & $0.28 \pm 0.18$ & $0.55 \pm 0.17$ & $0.40 \pm 0.24$ \\
\hline & 3 months & $0.64 \pm 0.11$ & $0.85 \pm 0.14$ & $0.77 \pm 0.22$ & $0.60 \pm 0.24$ \\
\hline
\end{tabular}

Table 3. Mean ( \pm standard deviation) dROMs, PAT, SAT, $\mathrm{HbA}_{1} \mathrm{c}$ outcome measures at baseline, one month and three months.

\begin{tabular}{|c|c|c|c|c|c|}
\hline Variable & Time point & Overall & \multicolumn{3}{|c|}{ Treatment Group } \\
\hline & & & Clhorhexidine & Ozone & Antioxidant \\
\hline & & Mean \pm SD & Mean \pm SD & Mean \pm SD & Mean \pm SD \\
\hline dROMs & & & & & \\
\hline & Baseline & $353 \pm 20.3$ & $396 \pm 37.4$ & $339 \pm 31.3$ & $310 \pm 22.4$ \\
\hline & 1 month & $332 \pm 17.0$ & $372 \pm 33.7$ & $309 \pm 23.1$ & $312 \pm 23.8$ \\
\hline & 3 months & $295 \pm 11.8$ & $263 \pm 14.1$ & $324 \pm 14.9$ & $285 \pm 33.9$ \\
\hline & & & & & \\
\hline & Baseline & $1838 \pm 173$ & $2113 \pm 389$ & $1547 \pm 217$ & $2009 \pm 144$ \\
\hline & 1 month & $1670 \pm 143$ & $1607 \pm 275$ & $1864 \pm 197$ & $1344 \pm 300$ \\
\hline & 3 months & $1502 \pm 152$ & $1039 \pm 185$ & $2058 \pm 89.6$ & $1060 \pm 377$ \\
\hline & & & & & \\
\hline & Baseline & $2083 \pm 360$ & $1757 \pm 241$ & $2347 \pm 636$ & $2059 \pm 1174$ \\
\hline & 1 month & $1071 \pm 215$ & $1673 \pm 497$ & $827 \pm 696$ & $569 \pm 179$ \\
\hline & 3 months & $1337 \pm 170$ & $1078 \pm 179$ & $1325 \pm 278$ & $1819 \pm 477$ \\
\hline HbA $_{1} \mathrm{c}$ & & & & & \\
\hline & Baseline & $6.92 \pm 0.22$ & $6.63 \pm 0.27$ & $7.27 \pm 0.32$ & $7.10 \pm 1.10$ \\
\hline & 3 months & $6.63 \pm 0.16$ & $6.41 \pm 0.25$ & $6.68 \pm 0.06$ & $6.80 \pm 0.80$ \\
\hline
\end{tabular}

Three months after treatment, dROMs and SAT changed significantly ( $\mathrm{p}$ value $<0.05$ ) as reported in table 4 . PAT remained unaltered in a range of deficiency. The oxidative balance of the entire cohort improved significantly three months after non-surgical periodontal treatment. Anyway, none of the tests applied reached statistically significant $p$-values for the whole-plot factor (group of treatment) indicating that the null hypothesis of no-treatment-effect on the oxidative balance could not be rejected. Ozone group showed the best results in terms of oxidative stress reduction. The laboratory analysis performed at the Department of Diabetology of the University of Pisa showed a mean value of $\mathrm{HbA}_{1} \mathrm{C}$ of $6.92 \%$ for the entire cohort at baseline. Three months after treatment, the mean value of $\mathrm{HbA}_{1} \mathrm{c}$ was $6.63 \%$. The reduction was statistically significant ( $p$-value $<0.05)$ three months after periodontal treatment (Table 4). Anyway, it was not possible to demonstrate a treatment effect in reducing glycated hemoglobin neither a different outcome between type I and type II diabetic patients.

\section{Discussion}

The present study was a randomized clinical investigation and it was based on emerging data that relate oxidative stress to both Diabetes and Periodontal disease [21]. The results of the present study 
Table 4. Longitudinal analysis for non-parametric distributions.

\begin{tabular}{|c|c|c|c|}
\hline \multicolumn{5}{|c|}{ p-value } \\
\hline Variable & Time effect & DM type effect & Treatment effect \\
\hline PPD & $<\mathbf{0 . 0 5}$ & 0.416 & 0.887 \\
\hline PI & $<\mathbf{0 . 0 5}$ & 0.821 & 0.839 \\
\hline$\%$ BoP & $<\mathbf{0 . 0 5}$ & 0.259 & 0.730 \\
\hline dROMs & $<\mathbf{0 . 0 5}$ & 0.157 & 0.579 \\
\hline PAT & $>0.05$ & 0.319 & 0.203 \\
\hline SAT & $<\mathbf{0 . 0 5}$ & 0.257 & 0.768 \\
\hline HbA $_{1 \mathrm{cc}}$ & $<\mathbf{0 . 0 5}$ & 0.764 & 0.325 \\
\hline
\end{tabular}

agreed with the current literature about the capability of non-surgical periodontal treatment to reduce systemic inflammation [22]. It has been demonstrated that chronic inflammation following periodontal infection may enhance the release of inflammation mediators and free radicals, thus threatening the natural history of diabetes [23]. In gingival tissues of subjects with diabetes who also have periodontitis, the presence of AGEs (advanced glycation end-products) and accompanying markers for increased oxidant stress have been demonstrated [24]. The present cohort of patients was characterized by its fair glycaemic control (mean value at baseline of $6.92 \%$ ) due to the fact that all the patients joined a strict regimen within the unit of Diabetology of the University of Pisa. The mean probing depth at baseline was $3.14 \mathrm{~mm}$ and the mean bleeding on probing was $76 \%$. The mean plaque index was 1.94 . These values pictured a mild-to-moderate periodontitis at baseline for the entire cohort. The descriptive statistics for the mean initial oxidative balance showed a tendency towards pathological ranges. The mean count of plasmatic free radicals (d-ROM) was 353 U.Carr., a value that denoted a moderate level of oxidative stress. At the same time, the plasmatic antioxidant status (PAT) showed a deficiency if compared to the general population. The amount of plasmatic reactive oxygen species (dROMs) showed a significant reduction three months after periodontal treatment in each of the three groups of treatment. The intergroup analysis did not highlight any remarkable differences in terms of dROMs reduction between the three treatment modalities. PAT values remained in a deficiency status at a three-month evaluation. The mean total salivary antioxidant capacity of the present cohort at baseline was 2083 U.Carr which resulted higher than normal range thus denoting a potential state of inflammation in diabetic patients affected by moderate periodontitis. The over-production of free radicals caused by periodontal inflammation is in fact supposed to cause an up-regulation of the anti-oxidant defence $[25,26]$. The results of the present study agree with literature relating high levels of salivary antioxidants (SAT) to a possible periodontal inflammatory condition. In response to oxidative stress, antioxidant enzymes appeared upregulated in inflamed periodontal tissues [27,28]. The longitudinal analysis of this trial showed a normalization of SAT values three months after non-surgical periodontal treatment. This inversion of tendency was coherent to the reduction of mean PPD, PI\% and BOP\% of the cohort during the follow-up. Since recently it has been hypothesized that oral dysbiosis might contribute to diabetes genesis among healthy individual [29], it would be crucial to explore new ways to monitor the oral microbiome behavior. The present clinical investigation has linked diabetes condition cross-sectionally to oxidative stress both systemic (plasmatic count) and local (salivary count). Periodontal treatment brought to periodontal indices normalization and to a reduction in terms of oxidative stress. Periodontal indexes showed a better outcome 1 month after treatment if compared to the three-month evaluation. This result highlighted how it is difficult to maintain an ideal balance within few months in problematic patients. In our opinion, it would be crucial to tailor the timing of hygiene recalls to each patient considering one's specific "periodontal deadline". Coming to glycaemic control, the mean reduction of glycated haemoglobin was 0.56 . It is a key point to note that baseline values of $\mathrm{HbA}_{1 \mathrm{c}}$ among this cohort were already almost acceptable for a group of diabetic patients. Therefore, a small reduction of $\mathrm{HbAlc}$ over three months, should not be ignored even if occurring within non-alarming values. At least, it would be worthwhile to investigate the same phenomenon over a larger sample including uncontrolled diabetic patients. The mean reduction of $\mathrm{HbAlc}$ was more evident among the ozone test group thus reinforcing the role of this particular type of adjunctive periodontal therapy in diabetic patients. The present study was designed as a pilot research in the field of perio-medicine. Given its limitations in terms of the number of patients and heterogeneity of the sample, it was impossible to establish any linear relationship between the variables of interest. The results obtained suggested the possibility to identify new markers describing the risk profile of diabetic patients to develop inflammatorybased-complications, periodontitis being included. Oxidative stress is an active factor both in the etiology and in the course of diabetes and periodontal disease. To monitor the oxidative balance of a complicated patient could mean preventing future major complications. It will be interesting in the future to further investigate the role of ozone therapy in the management of periodontal disease in diabetic patients.

\section{Disclosure statement}

The paper has been submitted solely to Journal of Dental, Oral and Craniofacial Research and that it is not concurrently under consideration for publication in another journal.

All individuals listed as authors agree that they have met the criteria of authorship and agree to the conclusions of the study.

\section{Conflicts of interest}

The authors report no conflicts of interest related to this study.

\section{Footnotes}

+ Aquolab s.r.l.EB2C Srl-Via Montevideo 13 - 20144 Milano - (Italy)

${ }^{*}$ Mectron s.p.a. via Loreto $15^{\circ} 16042$ Carasco (GE) Italy

${ }^{\complement} \mathrm{H} \& D$ s.r.l., Strada Langhirano ${ }^{\circ} 264 / 1^{\circ}$, Località Fontanini 43124 Parma (PR),Italy

\#Stata, StataCorp LP 4905 Lakeway Drive College Station, Texas 77845-4512 USA

${ }^{\circ} \mathrm{R}$ Studio free software, 250 Northern Ave, Boston, MA 02210

\section{Authors contribution}

Marconcini S: contributed to conception and design; contributed to acquisition, analysis, and interpretation; drafted manuscript; critically revised manuscript; gave final approval; agrees to be accountable for all aspects of work ensuring integrity and accuracy

Barone A: contributed to conception and design; contributed to analysis and interpretation; drafted manuscript; critically revised manuscript; gave final approval; agrees to be accountable for all aspects of work ensuring integrity and accuracy

Covani U: contributed to conception and design; contributed to analysis and interpretation; drafted manuscript; critically revised manuscript; gave final approval; agrees to be accountable for all aspects of work ensuring integrity and accuracy 
Genovesi AM: contributed to conception and design; contributed to acquisition and interpretation; drafted manuscript; critically revised manuscript; gave final approval; agrees to be accountable for all aspects of work ensuring integrity and accuracy

Soder B: contributed to conception and design; contributed to interpretation; drafted manuscript; critically revised manuscript; gave final approval; agrees to be accountable for all aspects of work ensuring integrity and accuracy

Giammarinaro E: contributed to conception and design; contributed to acquisition, analysis, and interpretation; drafted manuscript; critically revised manuscript; gave final approval; agrees to be accountable for all aspects of work ensuring integrity and accuracy

Giampietro O: contributed to conception and design; contributed to acquisition, analysis, and interpretation; drafted manuscript; critically revised manuscript; gave final approval; agrees to be accountable for all aspects of work ensuring integrity and accuracy

Giampietro C: contributed to conception and design; contributed to acquisition and analysis; drafted manuscript; critically revised manuscript; gave final approval; agrees to be accountable for all aspects of work ensuring integrity and accuracy

\section{References}

1. Page RC, Kornman KS (1997) The pathogenesis of human periodontitis: an introduction. Periodontol 2000 14: 9-11. [Crossref]

2. Hajishengallis G, Lamont RJ (2012) Beyond the red complex and into more complexity: the polymicrobial synergy and dysbiosis (PSD) model of periodontal disease etiology. Molecular oral microbiology 27: 409-419. [Crossref]

3. D'Aiuto F, Parkar M, Andreou G, Suvan J, Brett PM, et al. (2004) Periodontitis and systemic inflammation: control of the local infection is associated with a reduction in serum inflammatory markers. J Dent Res 2004; 83: 156-160. [Crossref]

4. Matthews JB, Wright HJ, Roberts A, Cooper PR, Chapple IL (2007) Hyperactivity and reactivity of peripheral blood neutrophils in chronic periodontitis. Clin Exp Immuno 147: 225-264. [Crossref]

5. Engelgau MM, Geiss LS, Saaddine JB, Boyle JP, Benjamin SM, et al. (2004) The evolving diabetes burden in the United States. Ann Intern Med 140: 945-950. [Crossref]

6. Novak MJ, Potter RM, Blodgett J, Ebersole JL (2008) Periodontal disease in Hispanic Americans with type 2 diabetes. J Periodontol 79: 629-636. [Crossref]

7. Kinane D, Bouchard P; Group E of European Workshop on Periodontology (2008) Periodontal diseases and health: consensus report of the sixth European workshop on periodontology. J Clin Periodontol 35: 333-337. [Crossref]

8. Awartani FA (2009) Evaluation of the relationship between type 2 diabetes and periodontal disease. Saudi Med J 30: 902-906. [Crossref]

9. Lockhart PB, Bolger AF, Papapanou PN, Osinbowale O, Trevisan M, et al. (2012) Periodontal disease and atherosclerotic vascular disease: does the evidence support an independent association? A scientific statement from the American Heart Association. Circulation 125: 2520-2544. [Crossref]

10. Engebretson S, Kocher T (2013) Evidence that periodontal treatment improves diabetes outcomes: a systematic review and meta-analysis. J Clin Periodontol 40: 153-169. [Crossref]

11. Friedewald VE, Kornman KS, Beck JD, Genco R, Goldfine A, et al. (2009) The American Journal of Cardiology and Journal of Periodontology editors' consensus: periodontitis and atherosclerotic cardiovascular disease. Am J Cardiol 80: 1021-1032. [Crossref]
12. Chapple IL, Genco R (2013) Diabetes and periodontal diseases: consensus report of the Joint EFP/AAP workshop on periodontitis and systemic diseases. $J$ Periodontol 84: 106-112. [Crossref]

13. Pussinen PJ, Tuomisto K, Jousilahti P, Havulinna AS, Sundvall J, et al. (2007) Endotoxemia, immune response to periodontal pathogens, and systemic inflammation associate with incident cardiovascular disease events. Arterioscler Thromb Vasc Biol 27: 1433-1439. [Crossref]

14. Nagata M (2005) Inflammatory cells and oxygen radicals. Curr Drug Targets Inflamm Allergy 4: 503-504. [Crossref]

15. Bullon P, Morillo JM, Ramirez-Tortosa MC, Quiles JL, Newman HN, et al. (2009) Metabolic syndrome and periodontitis: is oxidative stress a common link? J Dent Res 88: 503-518. [Crossref]

16. Hiramatsu K, Arimori S (1988) Increased superoxide production by mononuclear cells of patients with hypertriglyceridemia and diabetes. Diabetes 37: 832-837. [Crossref]

17. Sculley DV, Langley-Evans SC (2002) Salivary antioxidants and periodontal disease status. Proc Nutr Soc 61: 137-143. [Crossref]

18. Tonetti MS1, Claffey N; European Workshop in Periodontology group C (2005) Advances in the progression of periodontitis and proposal of definitions of a periodontitis case and disease progression for use in risk factor research. Group C consensus report of the 5th European Workshop in Periodontology. J Clin Periodontol 6: 210-213. [Crossref]

19. Brunner E, Langer F (2000) Nonparametric analysis of ordered categorical data in designs with longitudinal observations and small sample sizes. Biometrical J 42: 663-675.

20. Preshaw PM, Alba AL, Herrera D, Jepsen S, Konstantinidis A, et al. (2012) Periodontitis and diabetes: a two-way relationship. Diabetologia 55: 21-31. [Crossref]

21. Eke PI, Genco RJ (2007) CDC periodontal disease surveillance project: background, objectives, and progress report. J Periodontol 78: 1366-1371. [Crossref]

22. Karima M, Kantarci A, Ohira T, Hasturk H, Jones VL, et al. (2005) Enhanced superoxide release and elevated protein kinase $\mathrm{C}$ activity in neutrophils from diabetic patients: association with periodontitis. J Leukoc Biol 78: 862-870. [Crossref]

23. Abusleme L, Dupuy AK, Dutzan N, Silva N, Burleson JA, et al. (2013) The subgingival microbiome in health and periodontitis and its relationship with community biomass and inflammation. ISME J 7: 1016-1025. [Crossref]

24. Waddington RJ, Moseley R, Embery G (2000) Periodontal disease mechanisms reactive oxygen species: a potential role in the pathogenesis of periodontal diseases. Oral Dis 6: 138-151. [Crossref]

25. Akalin FA1, Isiksal E, Baltacioglu E, Renda N, Karabulut E (2008) Superoxide dismutase activity in gingiva in type-2 diabetes mellitus patients with chronic periodontitis. Arch Oral Biol 53: 44-52. [Crossref]

26. Gümüş P, Buduneli N, Cetinkalp S, Hawkins SI, Renaud D, et al. (2009) Salivary antioxidants in patients with type 1 or 2 diabetes and inflammatory periodontal disease: a case-control study. J Periodontol 80: 1440-1446. [Crossref]

27. Wei D, Zhang XL, Wang YZ, Yang CX, Chen G (2010) Lipid peroxidation levels, total oxidant status and superoxide dismutase in serum, saliva and gingival crevicular fluid in chronic periodontitis patients before and after periodontal therapy. Aust Dent $J$ 55: 70-78. [Crossref]

28. Trivedi S, Lal N, Mahdi AA, Mittal M, Singh B, et al. (2014) Evaluation of antioxidan enzymes activity and malondialdehyde levels in patients with chronic periodontitis and diabetes mellitus. J Periodontol 85: 713-720. [Crossref]

29. Demmer RT, Desvarieux M, Holtfreter B, Jacobs DR Jr, Wallaschofski H, et al. (2010) Periodontal status and $\mathrm{A} 1 \mathrm{C}$ change: longitudinal results from the study of health in pomerania (SHIP). Diabetes care 33: 1037-1043. [Crossref]

Copyright: (C2017 Marconcini S. This is an open-access article distributed under the terms of the Creative Commons Attribution License, which permits unrestricted use, distribution, and reproduction in any medium, provided the original author and source are credited. 